\title{
Proximity force approximation and specular reflection: Application of the WKB limit of Mie scattering to the Casimir effect
}

\author{
Benjamin Spreng,,${ }^{1,}$ Michael Hartmann, ${ }^{1}$ Vinicius Henning, ${ }^{2}$ Paulo A. Maia Neto, ${ }^{2}$ and Gert-Ludwig Ingold ${ }^{1, \dagger}$ \\ ${ }^{1}$ Institut für Physik, Universität Augsburg, 86135 Augsburg, Germany \\ ${ }^{2}$ Instituto de Física, Universidade Federal do Rio de Janeiro, CP 68528, Rio de Janeiro RJ 21941-909, Brazil
}

(Received 6 March 2018; published 13 June 2018)

\begin{abstract}
The electromagnetic Casimir interaction between two spheres is studied within the scattering approach using the plane-wave basis. It is demonstrated that the proximity force approximation (PFA) corresponds to the specularreflection limit of Mie scattering. Using the leading-order semiclassical WKB approximation for the direct reflection term in the Debye expansion for the scattering amplitudes, we prove that PFA provides the correct leading-order term in the small-distance limit for arbitrary materials and temperatures in the sphere-sphere and the plane-sphere geometry. Our derivation implies that only a small section around the points of closest approach between the interacting spherical surfaces contributes in the PFA regime. The corresponding characteristic length scale is estimated from the width of the Gaussian integrand obtained within the saddle-point approximation. At low temperatures, the area relevant for the thermal corrections is much larger than the area contributing to the zero-temperature result.
\end{abstract}

DOI: 10.1103/PhysRevA.97.062504

\section{INTRODUCTION}

In his seminal work, Casimir analyzed the effect of fluctuation-induced phenomena [1,2] on plane-parallel mirrors finding an attractive force. The geometry originally studied by Casimir is rarely realized in experiments [3,4]. Instead most experiments probe the Casimir interaction between a spherical and a planar surface in order to minimize systematic errors (see, e.g., Refs. [5-11] for recent experiments and [12-14] for reviews of earlier ones). In addition, the sphere-sphere geometry has lately gained experimental interest $[15,16]$.

On the theoretical side, the scattering approach [17-19] allows one to compute the Casimir energy from the scattering matrices of the individual bodies interacting across a region of empty space. It also provides a clear physical picture of the Casimir effect as resulting from the reverberation or multiple scattering of vacuum or thermal electromagnetic field fluctuations between the interacting surfaces [20,21].

These theoretical and experimental advances were disconnected until very recently, when exact numerical results for typical experimental conditions were obtained from the scattering approach using a symmetrized round-trip operator $[22,23]$. Furthermore, a numerically less demanding approximate semianalytic formula based on the exact high-temperature expression for Drude metals was proposed [24]. Instead of applying these techniques, the surface curvature in real experiments is typically taken into account with the help of the proximity force (Derjaguin) approximation (PFA) [25], in which the result for the Casimir energy between parallel plates is averaged over the local distances corresponding to the geometry of interest. PFA is also often employed in surface science [26], for instance, in the comparison with experimental results for the van der Waals interaction between spherical colloids [27,28].

\footnotetext{
*benjamin.spreng@physik.uni-augsburg.de

† gert.ingold@physik.uni-augsburg.de
}

The PFA approach to spherical curvature is conceptually different from the picture of electromagnetic field reverberation between spherical surfaces that results from the scattering approach. Nevertheless, we establish in this paper a direct connection between the two approaches. In the limit of small distances between the interacting surfaces, the dominant contribution to the multiple scattering between the surfaces is shown to result from semiclassical WKB specular reflection by a small section of the sphere's surface around the point of closest approach. The final expression for the Casimir force then coincides with the PFA result.

The connection between the scattering approach and PFA was first analyzed in the context of the roughness correction to the Casimir energy for parallel planes. The PFA result for the roughness correction was derived from the more general case in the limit of short distances and smooth surfaces by considering the value of the perturbative kernel at zero momentum [29]. This line of reasoning can be generalized to the entire perturbative series [30] within the derivative expansion approach [31], although it is not possible to derive explicit results for all the corresponding kernels in this case. Nevertheless, the PFA result is obtained in leading order for surfaces that can be continuously deformed from the planar symmetry, as long as the kernel functions have a well-defined limit at zero momentum [30]. Since the first condition does not hold for compact objects, this derivation does not constitute a proof of PFA for the plane-sphere nor for the sphere-sphere geometries, even though the leading-order correction to PFA has been successfully derived [32-34] whenever the perturbative kernel is analytical at zero momentum [35].

For the plane-sphere setup, the validity of PFA in the shortdistance limit was shown at zero temperature for both perfect [36,37] and real metals [38] by developing the scattering approach in the multipolar basis and taking suitable asymptotic approximations. Earlier results were derived for a scalar field model at zero temperature $[39,40]$. In the opposite limit of high 


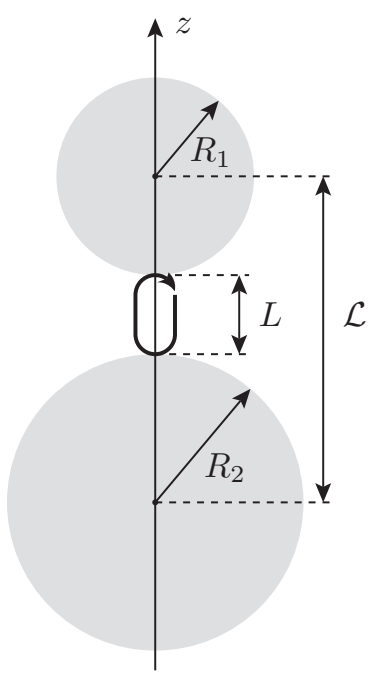

FIG. 1. Scattering geometry consisting of two spheres with radii $R_{1}$ and $R_{2}$ and a surface-to-surface distance $L$. The distance between the spheres' centers is $\mathcal{L}=R_{1}+R_{2}+L$. The round trip discussed in the text is displayed between the two spheres.

temperatures, a similar derivation was recently undertaken for perfect metals [41]. Reference [42] derived an exact formula, also compatible with the PFA leading-order result, for Drude metals in the high-temperature limit by using bispherical multipoles.

Here, we consider the general case of arbitrary temperatures and materials. Our setup consists of two spheres with radii $R_{1}$ and $R_{2}$ in empty space, at a distance of closest approach $L$, as indicated in Fig. 1. The center-to-center distance is $\mathcal{L}=L+$ $R_{1}+R_{2}$ along the $z$ axis. The plane-sphere case is obtained at any step of our derivation by taking the radius of one sphere to infinity.

The sphere-sphere Casimir interaction has been analyzed within the scattering approach for large and moderate distances $[15,18,43,44]$. In addition, corrections to PFA were obtained analytically for idealized boundary conditions at zero temperature [45], and numerically for more general situations [34]. Commonly, the scattering formula is expanded in terms of angular momentum multipolar waves, which is particularly natural for not too small distances. In this paper, we show that the linear momentum representation is better adapted to the short-distance PFA regime $L \ll R_{1}, R_{2}$ because of its direct connection to the physical picture of specular reflection between the spherical surfaces.

Our derivation brings into light the main physical ingredients underlying the PFA regime. We show that only the direct reflection term in the Debye expansion [46] of the Mie scattering matrix contributes. Moreover, this contribution is taken in the semiclassical WKB approximation, which has a direct physical interpretation in terms of specular reflection by the sphere's surface [47]. More importantly, the multiple scattering between the surfaces defines a scale for the variation of the momentum component parallel to the $x-y$ plane. This scale will allow us to specify the spherical cap on the spheres that actually contributes in the PFA regime.

Our semiclassical WKB derivation in the momentum representation should not be confused with the semiclassical treatments of the Casimir interaction in the position representation [48-50]. The standard Gutzwiller trace formula fails badly in the PFA regime because the relevant surface area increases as one approaches this limit [49]. In other words, the semiclassical PFA limit cannot be connected to periodic orbits obtained from a stationary-phase approximation in the position representation, because position is poorly resolved in this limit. On the other hand, the condition of specular reflection makes the integration range for the conjugate momentum variable increasingly narrow as the distance between the spheres becomes very small compared to their radii, allowing us to obtain the PFA result from a saddle-point approximation for the scattering formula.

The paper is organized as follows. Section II presents the general development of the scattering formula for the Casimir free energy in the plane-wave basis. The following sections apply this formalism to the case of two spheres in the shortdistance limit. The Mie scattering matrix elements in the planewave basis, for arbitrary directions of incidence, are presented in Sec. III. The corresponding WKB approximation is derived in Sec. IV and Sec. V calculates the resulting Casimir free energy in the saddle-point approximation. Section VI presents an estimation of the area on the spheres that actually contributes in the short-distance limit. Concluding remarks are presented in Sec. VII and the appendixes contain some additional technical details.

\section{CASIMIR FREE ENERGY IN THE PLANE-WAVE BASIS}

In a homogeneous medium, the electric and the magnetic field satisfy the vector Helmholtz equation. A convenient basis set consists of plane waves characterized by a wave vector $\mathbf{K}$ and the polarization $p$. In order to define the polarization basis, we assume a given $z$ axis which can be appropriately fixed later on and choose an incidence plane spanned by the $z$ axis and the wave vector $\mathbf{K}$. Denoting unit vectors by a hat, we obtain the basis vectors for transverse electric (TE) and transverse magnetic (TM) modes as

$$
\hat{\boldsymbol{\epsilon}}_{\mathrm{TE}}=\frac{\hat{\mathbf{z}} \times \hat{\mathbf{K}}}{|\hat{\mathbf{z}} \times \hat{\mathbf{K}}|}, \quad \hat{\boldsymbol{\epsilon}}_{\mathrm{TM}}=\hat{\boldsymbol{\epsilon}}_{\mathrm{TE}} \times \hat{\mathbf{K}} .
$$

Thus the TE polarization is perpendicular to the incidence plane while the TM polarization lies in it.

As we will see later in this section, it is convenient to fix the frequency which by means of the dispersion relation is obtained as $\omega=c|\mathbf{K}|$ with $c$ being the speed of light. It is then sufficient to specify the projection $\mathbf{k}=\left(K_{x}, K_{y}, 0\right)$ of the wave vector $\mathbf{K}$ onto the $x-y$ plane perpendicular to the $z$ axis. In order to uniquely define the wave vector, we finally need to fix the direction of the propagation in the $z$ direction by

$$
K_{z}=\phi k_{z}, \quad k_{z} \equiv\left(\omega^{2} / c^{2}-\mathbf{k}^{2}\right)^{1 / 2},
$$

with $\phi= \pm 1$. We thus arrive at the angular spectral representation expressing the plane-wave basis as $\{|\omega, \mathbf{k}, p, \phi\rangle\}$ [51]. In position space, the basis functions read

$$
\langle x, y, z \mid \omega, \mathbf{k}, p, \phi\rangle=\hat{\boldsymbol{\epsilon}}_{p}\left(\frac{1}{2 \pi}\left|\frac{\omega}{c k_{z}}\right|\right)^{1 / 2} e^{i\left(\mathbf{k r}+\phi k_{z} z\right)},
$$

where $\mathbf{r}=(x, y, 0)$. The normalization factor is appropriate for the angular spectral representation where the integration 
is performed over the frequency and the projection of the wave vector into the $x-y$ plane.

For the purpose of this section, we consider two arbitrary objects that can be separated by a plane parallel to the $x-y$ plane. The reference point of object 1 is located in the origin and the reference point of object 2 is located at $z=\mathcal{L}$. The starting point of our analysis is the scattering approach to the Casimir effect in imaginary frequencies $\xi=i \omega$, where the free energy is expressed as $[17,18]$

$$
\mathcal{F}=\frac{k_{\mathrm{B}} T}{2} \sum_{n=-\infty}^{\infty} \operatorname{tr} \log \left[1-\mathcal{M}\left(\left|\xi_{n}\right|\right)\right]
$$

in terms of a sum over the Matsubara frequencies $\xi_{n}=$ $2 \pi n k_{\mathrm{B}} T / \hbar$. The central object here is the round-trip operator

$$
\mathcal{M}=\mathcal{R}_{1} \mathcal{T}_{12} \mathcal{R}_{2} \mathcal{T}_{21}
$$

describing a complete round trip of an electromagnetic wave between the two scatterers in the order indicated in Fig. 1. $\mathcal{R}_{j}$ denotes the reflection operator at object $j=1,2$ and $\mathcal{T}_{21}$ describes a translation from the reference frame of object 1 to the reference frame of object 2 , and vice versa for $\mathcal{T}_{12}$.

In the plane-wave basis the translation operators are diagonal with matrix elements $e^{-\kappa \mathcal{L}}$, where $\kappa=\left(\xi^{2} / c^{2}+k^{2}\right)^{1 / 2}$ denotes the $z$ component of the wave vector associated with the imaginary frequency $\xi$. As the frequency $\xi$ remains constant during a round trip, we suppress the notation $\xi$ in the labeling of the basis elements.

The logarithm in (4) can be expanded in a Mercator series

$$
\mathcal{F}=-\frac{k_{\mathrm{B}} T}{2} \sum_{n=-\infty}^{\infty} \sum_{r=1}^{\infty} \frac{1}{r} \operatorname{tr} \mathcal{M}^{r}\left(\left|\xi_{n}\right|\right)
$$

and the trace of the $r$ th power of the round-trip operator is given by

$$
\begin{aligned}
\operatorname{tr} \mathcal{M}^{r}= & \sum_{p_{1}, \ldots, p_{2 r}} \int \frac{d \mathbf{k}_{1} \ldots d \mathbf{k}_{2 r}}{(2 \pi)^{4 r}} \prod_{j=1}^{r} e^{-\left(\kappa_{2 j}+\kappa_{2 j-1}\right) \mathcal{L}} \\
& \times\left\langle\mathbf{k}_{2 j+1}, p_{2 j+1},-\left|\mathcal{R}_{1}\right| \mathbf{k}_{2 j}, p_{2 j},+\right\rangle \\
& \times\left\langle\mathbf{k}_{2 j}, p_{2 j},+\left|\mathcal{R}_{2}\right| \mathbf{k}_{2 j-1}, p_{2 j-1},-\right\rangle .
\end{aligned}
$$

Here, we have used the convention of cyclic indices $p_{2 r+1} \equiv$ $p_{1}$ and $\mathbf{k}_{2 r+1} \equiv \mathbf{k}_{1}$. Equations (6) and (7) can be interpreted as an expansion in round trips. The free energy consists of contributions from a single round trip within the cavity, up to infinitely large numbers of round trips. Also, the expansion in round trips is a natural way to compute $\operatorname{tr} \log (1-\mathcal{M})$ of a nondiagonal round-trip operator $\mathcal{M}$ expressed in a continuous basis.

The exponential factor in (7) might suggest that the component in $z$ direction $\kappa$ of the imaginary wave vectors is constrained to values of the order of $1 / \mathcal{L}$. However, we will see later for the special case of the sphere-sphere configuration that the reflection matrix elements grow exponentially with the size of the corresponding objects. As a consequence, much larger values for $\kappa$ of the order of $1 / L$ are possible, where $L$ denotes the closest distance between the two objects.

While our discussion so far was fairly general, we will specialize on the geometry of two spheres in the following sections.

\section{SCATTERING AT A SPHERE}

While the translation operators in the plane-wave basis are trivial, the reflection operators require more care. For scattering at a single sphere, we can make the scattering plane spanned by the initial and the reflected wave vectors coincide with the incidence plane defining the polarizations as explained in Sec. II. In the sphere-sphere setup shown in Fig. 1, however, the $z$ axis is defined by the centers of the two spheres. Then, in general, the scattering plane and the incidence plane will not coincide and one has to change the polarization basis as explained in Appendix A. Nevertheless, it will turn out that the results can mostly be cast in quantities familiar from the standard Mie theory, allowing us to relate PFA to the concepts of geometrical optics.

Then, the matrix elements of the reflection operator $\mathcal{R}$ at a sphere are given by

$$
\begin{aligned}
\left\langle\mathbf{k}_{j}, \mathrm{TM}|\mathcal{R}| \mathbf{k}_{i}, \mathrm{TM}\right\rangle & =\frac{2 \pi c}{\xi \kappa_{j}}\left[A S_{2}(\Theta)+B S_{1}(\Theta)\right], \\
\left\langle\mathbf{k}_{j}, \mathrm{TE}|\mathcal{R}| \mathbf{k}_{i}, \mathrm{TE}\right\rangle & =\frac{2 \pi c}{\xi \kappa_{j}}\left[A S_{1}(\Theta)+B S_{2}(\Theta)\right], \\
\left\langle\mathbf{k}_{j}, \mathrm{TM}|\mathcal{R}| \mathbf{k}_{i}, \mathrm{TE}\right\rangle & =-\frac{2 \pi c}{\xi \kappa_{j}}\left[C S_{1}(\Theta)+D S_{2}(\Theta)\right], \\
\left\langle\mathbf{k}_{j}, \mathrm{TE}|\mathcal{R}| \mathbf{k}_{i}, \mathrm{TM}\right\rangle & =\frac{2 \pi c}{\xi \kappa_{j}}\left[C S_{2}(\Theta)+D S_{1}(\Theta)\right],
\end{aligned}
$$

where the prefactor results from the normalization within the angular spectral representation. Here, we have omitted the value of $\phi$ which should be different for the two waves involved in a matrix element. In addition, the signs of $C$ and $D$ depend on the direction of propagation as specified in Eq. (A8). The Mie scattering amplitudes for polarizations perpendicular and parallel to the scattering plane are given by [52]

$$
\begin{aligned}
& S_{1}(\Theta)=\sum_{\ell=1}^{\infty} \frac{2 \ell+1}{\ell(\ell+1)}\left[a_{\ell} \pi_{\ell}(\cos (\Theta))+b_{\ell} \tau_{\ell}(\cos (\Theta))\right], \\
& S_{2}(\Theta)=\sum_{\ell=1}^{\infty} \frac{2 \ell+1}{\ell(\ell+1)}\left[a_{\ell} \tau_{\ell}(\cos (\Theta))+b_{\ell} \pi_{\ell}(\cos (\Theta))\right],
\end{aligned}
$$

respectively. The scattering angle is defined relative to the forward direction (cf. Fig. 2) and, for imaginary frequencies, is given by

$$
\cos (\Theta)=-\frac{c^{2}}{\xi^{2}}\left(\mathbf{k}_{i} \cdot \mathbf{k}_{j}+\kappa_{i} \kappa_{j}\right) .
$$

The angular functions $\pi_{\ell}$ and $\tau_{\ell}$ are defined by [52]

$$
\begin{aligned}
\pi_{\ell}(z) & =P_{\ell}^{\prime}(z), \\
\tau_{\ell}(z) & =-\left(1-z^{2}\right) P_{\ell}^{\prime \prime}(z)+z P_{\ell}^{\prime}(z),
\end{aligned}
$$

with the Legendre polynomials $P_{\ell}$ and the prime denoting a derivative with respect to the argument $z$.

The coefficients $A, B, C$, and $D$ are functions of $\mathbf{k}_{i}$ and $\mathbf{k}_{j}$. Explicit expressions are derived in Appendix A and given in (A8). The Mie coefficients $a_{\ell}$ and $b_{\ell}$ [52] represent the partial wave electric and magnetic multipole scattering amplitudes, respectively, for an isotropic sphere. They depend on the electromagnetic response of the sphere material. For 


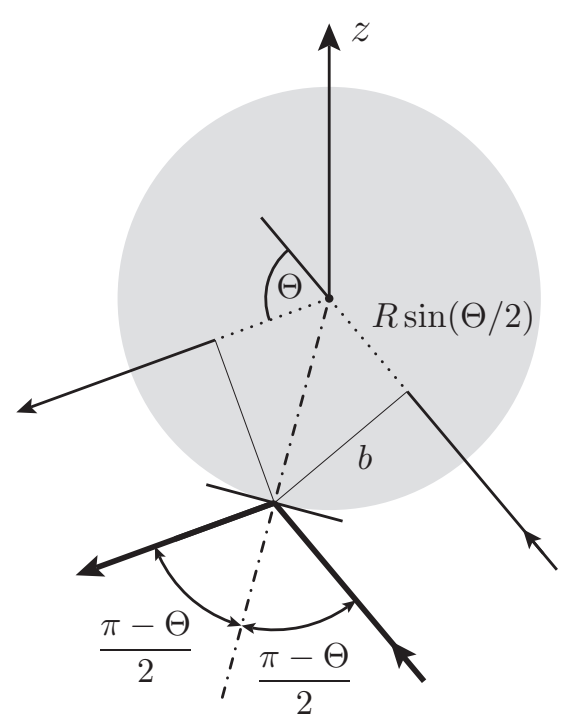

FIG. 2. Geometrical optics limit for the direct reflection by a sphere of radius $R$. Within the WKB approximation, a given scattering angle $\Theta$ defines the impact parameter $b=R \cos (\Theta / 2)$. Seen from the tangent plane to the sphere, the angle of incidence is given by $(\pi-\Theta) / 2$. The missing phase of a ray with frequency $\omega$ reflected on the sphere's surface with respect to a corresponding ray passing via the sphere's center before being deflected amounts to $2(\omega R / c) \sin (\Theta / 2)$.

simplicity, we restrict ourselves to homogeneous nonmagnetic spheres in the following.

\section{WKB APPROXIMATION}

In order to obtain PFA as the leading asymptotics for large radii $R_{1}, R_{2} \gg L$, asymptotic expressions for the matrix elements are required. For this purpose, it is convenient to write the scattering amplitudes by means of the Debye expansion [47], i.e., a decomposition into an infinite series of terms representing multiple internal reflections. In the limit of a large radius $R$, the direct reflection term of the Debye expansion gives the main contribution since the phase factor acquired by propagation inside the spheres yields exponentially small terms when considering the imaginary frequency domain.

For real frequencies and large size parameters $\omega R / c \gg 1$, the asymptotic expression for the direct reflection term has been derived from the WKB approximations for the Mie coefficients and angular functions by taking the saddle-point approximation for the integral over angular momenta [46]. The resulting expression is valid for all scattering directions except near the forward one, which is not relevant for the Casimir interaction. In fact, the Casimir energy as given by (6) and (7) is obtained from round trips containing only backward scattering channels. Instead of real frequencies, we need the asymptotics for imaginary frequencies $\xi$, for which a very similar WKB derivation can be performed. The resulting expression coincides with the one obtained for real frequencies $\omega$ after replacing $\omega \rightarrow i \xi$. The leading asymptotics of the scattering amplitude is then given by

$$
S_{p}(\Theta) \simeq \frac{1}{2}(\xi R / c) r_{p}((\pi-\Theta) / 2) e^{2(\xi R / c) \sin (\Theta / 2)},
$$

with $p=1,2$ corresponding to TE and TM modes, respectively. $r_{\mathrm{TE}}$ and $r_{\mathrm{TM}}$ are the familiar Fresnel reflection coefficients [53] for a wave in vacuum impinging at an angle of incidence $\theta$ on a medium with permittivity $\varepsilon$,

$$
\begin{aligned}
r_{\mathrm{TE}}(\theta) & =\frac{\cos (\theta)-\sqrt{\varepsilon-\sin ^{2}(\theta)}}{\cos (\theta)+\sqrt{\varepsilon-\sin ^{2}(\theta)}}, \\
r_{\mathrm{TM}}(\theta) & =\frac{\varepsilon \cos (\theta)-\sqrt{\varepsilon-\sin ^{2}(\theta)}}{\varepsilon \cos (\theta)+\sqrt{\varepsilon-\sin ^{2}(\theta)}} .
\end{aligned}
$$

The asymptotics of the scattering amplitudes can be understood in terms of geometrical optics in the real frequency domain [54]. For a given scattering angle $\Theta$, the main contribution to $S_{1}$ and $S_{2}$ in Eq. (9) comes from the neighborhood of the angular momentum value $\ell=(\omega R / c) \cos (\Theta / 2)$ [54]. In the semiclassical approximation, the localization principle [47] connects waves with angular momentum $\ell \gg 1$ to localized rays defining an impact parameter $b=(c / \omega) \ell$. Thus the derivation of the WKB approximation (12) defines rays corresponding to the impact parameter $b=R \cos (\Theta / 2)$ shown in Fig. 2 . Such rays hit the sphere surface with an incidence angle of $(\pi-\Theta) / 2$, which is precisely the value required for obtaining the scattering angle $\Theta$ from the condition of specular reflection at the tangent plane indicated in the figure. Comparing the reflection at the tangent plane (thick lines) and at the sphere with its center as reference point, one finds a difference in path length amounting to $2(\omega R / c) \sin (\Theta / 2)$. In this way, the last two factors of (12) find their natural explanation. The first factor is responsible for providing the correct scattering cross section proportional to $R^{2}$.

The asymptotics of the Mie scattering amplitudes (12) does not cover the zero frequency case, which is required in the Matsubara sum. In Appendix B, we show that the scattering amplitudes for $\xi=0$ coincide with the scattering amplitudes at finite imaginary frequencies (12) evaluated at $\xi=0$.

In order to derive the leading asymptotic expression for the scattering matrix elements (8), we make use of (12) to obtain

$$
\left\langle\mathbf{k}_{j}, p_{j}|\mathcal{R}| \mathbf{k}_{i}, p_{i}\right\rangle \simeq \frac{\pi R}{\kappa_{j}} e^{2(\xi R / c) \sin (\Theta / 2)} \rho_{p_{j}, p_{i}},
$$

with

$$
\begin{aligned}
\rho_{\mathrm{TM}, \mathrm{TM}} & =A r_{\mathrm{TM}}+B r_{\mathrm{TE}}, \\
\rho_{\mathrm{TE}, \mathrm{TE}} & =A r_{\mathrm{TE}}+B r_{\mathrm{TM}}, \\
\rho_{\mathrm{TM}, \mathrm{TE}} & =-C r_{\mathrm{TE}}-D r_{\mathrm{TM}}, \\
\rho_{\mathrm{TE}, \mathrm{TM}} & =C r_{\mathrm{TM}}+D r_{\mathrm{TE}} .
\end{aligned}
$$

The WKB expression (14) for the reflection matrix element already indicates an exponential growth with the sphere radius $R$ as anticipated in the discussion at the end of Sec. II.

\section{PFA FROM SADDLE-POINT APPROXIMATION}

We will now derive the proximity force approximation based on the scattering matrix elements (14) obtained within the WKB approximation. The main step consists in evaluating within the saddle-point approximation the trace over $\mathcal{M}^{r}$ appearing in the expansion (6) of the free energy. This approach 
requires large sphere radii $R_{1}, R_{2} \gg L$ (cf. Fig. 1), a limit in which PFA is expected to hold. While we will carry out the calculation for the sphere-sphere geometry, we will briefly comment on the limit of the plane-sphere geometry when appropriate.

\section{A. Round trips within WKB approximation}

The main quantity in the round-trip expansion of the free energy (6) is the trace over the $r$ th power of the round-trip operator, which in the plane-wave representation is given by (7). After inserting the WKB scattering matrix elements (14) and employing polar coordinates $\left(k_{i}, \varphi_{i}\right)$ in the $x-y$ plane, we express the result in a form suitable for the saddle-point approximation as

$$
\begin{aligned}
\operatorname{tr} \mathcal{M}^{r} \simeq & \int_{0}^{\infty} d^{2 r} k \int_{0}^{2 \pi} d^{2 r} \varphi g\left(\mathbf{k}_{1}, \ldots, \mathbf{k}_{2 r}\right) \\
& \times e^{-\left(R_{1}+R_{2}\right) f\left(\mathbf{k}_{1}, \ldots, \mathbf{k}_{2 r}\right)} .
\end{aligned}
$$

The symbol $\simeq$ indicates that the result is only valid in the small distance limit $L \ll R_{1}, R_{2}$. Here, we have used $R_{1}+R_{2}$ as a large parameter for the saddle-point approximation. Another choice, e.g., an individual radius, would equally be possible and would yield the same final result.

In (16) we introduced the function

$$
\begin{aligned}
& g\left(\mathbf{k}_{1}, \ldots, \mathbf{k}_{2 r}\right) \\
& =\left(\frac{R_{1} R_{2}}{16 \pi^{2}}\right)^{r} \sum_{p_{1}, \ldots, p_{2 r}} \prod_{j=1}^{r} \frac{k_{2 j} k_{2 j-1}}{\kappa_{2 j} \kappa_{2 j-1}} \rho_{p_{2 j+1}, p_{2 j}}^{(1)}\left(\mathbf{k}_{2 j+1}, \mathbf{k}_{2 j}\right) \\
& \quad \times \rho_{p_{2 j}, p_{2 j-1}}^{(2)}\left(\mathbf{k}_{2 j}, \mathbf{k}_{2 j-1}\right) e^{-\left(\kappa_{2 j}+\kappa_{2 j-1}\right) L},
\end{aligned}
$$

where the superscript of the factors $\rho$ defined in (15) indicates the sphere for which the Fresnel reflection coefficient is to be taken.

The function in the exponent of (16) is given by

$$
f\left(\mathbf{k}_{1}, \ldots, \mathbf{k}_{2 r}\right)=\frac{1}{R_{1}+R_{2}} \sum_{j=1}^{r}\left(R_{1} \eta_{2 j}+R_{2} \eta_{2 j-1}\right),
$$

where the terms with even and odd indices are contributions from sphere 1 and 2, respectively, and

$\eta_{i}=\kappa_{i}+\kappa_{i+1}-\sqrt{2\left[(\xi / c)^{2}+\kappa_{i} \kappa_{i+1}+k_{i} k_{i+1} \cos \left(\varphi_{i}-\varphi_{i+1}\right)\right]}$.

While the last term on the right-hand side arises from the phase shift illustrated in Fig. 2, the first two terms are associated with a translation over twice the radius of the sphere at which the reflection occurs. As a consequence, the last factor in (17) only depends on the closest distance $L$ between the two spheres.

\section{B. Saddle-point manifold}

In order to evaluate the $4 r$ integrals in (16) within the saddlepoint approximation, we need to determine the stationary points. In fact, there exists a two-dimensional manifold of saddle points

$$
k_{1}=\cdots=k_{2 r}=k_{*}, \quad \varphi_{1}=\cdots=\varphi_{2 r}=\varphi_{*}
$$

parametrized by $k_{*}$ and $\varphi_{*}$. Thus, on the saddle-point manifold, the change of angle $\varphi=\varphi_{j+1}-\varphi_{j}$ vanishes and leads to a significant simplification because then the incidence and scattering planes coincide. As a consequence, $A=1, B=$ $C=D=0$ as can be verified also from the relations (A8) by setting $\varphi=0$. Thus, in view of (15), the polarization is always conserved during the scattering processes within the saddle-point approximation. The trace over $r$ round-trip operators (16) can now be decomposed into two independent polarization contributions

$$
\operatorname{tr} \mathcal{M}^{r}=\operatorname{tr} \mathcal{M}_{\mathrm{TE}}^{r}+\operatorname{tr} \mathcal{M}_{\mathrm{TM}}^{r} .
$$

The saddle-point manifold (20) also implies that the projection of the wave vector onto the $x-y$ plane is conserved during the reflection. Within the WKB approximation, this is the case when in Fig. 2 the tangent plane on which the reflection occurs was perpendicular to the $z$ axis. Under this condition, the WKB phase shift upon reflection $2(\xi R / c) \sin (\Theta / 2)$ can be expressed as $2 \kappa R$. This precisely cancels the term arising from the translation by twice the sphere radius. As a consequence the exponent (18) vanishes on the saddle-point manifold,

$$
\left.f\right|_{\text {S.P. }}=0 .
$$

For the prefactor in the integrand of (16), we now obtain for the two polarization contributions $p=\mathrm{TE}, \mathrm{TM}$

$$
\left.g_{p}\right|_{\mathrm{S} . \mathrm{P} .}\left(k_{*}\right)=\left(\frac{R_{1} R_{2}}{16 \pi^{2}} \frac{k_{*}^{2}}{\kappa_{*}^{2}} r_{p}^{(1)} r_{p}^{(2)}\right)^{r} e^{-2 r \kappa_{*} L}
$$

on the saddle-point manifold. Here, we have introduced $\kappa_{*}=$ $\left(\xi^{2} / c^{2}+k_{*}^{2}\right)^{1 / 2}$. The Fresnel coefficients (13) are evaluated at the angle

$$
\theta=\arccos \left(\kappa_{*} c / \xi\right) .
$$

On the saddle-point manifold, only a translation by $L$ remains, which just corresponds to the distance between the two tangent planes perpendicular to the $z$ axis facing each other. Thus the relevant length scale for the $z$ component of the imaginary wave vector is given by $L$ instead of $\mathcal{L}$ as (7) had seemed to imply.

The result (23) might raise some concerns about a divergence associated with the plane-sphere limit. Choosing without loss of generality $R_{1} \leqslant R_{2}$, the plane-sphere limit reads $R_{2} \rightarrow \infty$. We know that the wave vector $\mathbf{k}$ is conserved during a reflection at the plane, so that $2 r$ integrations have to drop out in this limit. In fact, we will see in the next subsection that for $R_{2} \rightarrow \infty$ indeed $2 r$ delta functions appear for which the factor $R_{2} / 4 \pi$ contained in (23) provides the normalization.

\section{Hessian matrix}

We now turn to the Hessian matrix $\mathrm{H}$ of the function $f$ (18) evaluated on the saddle-point manifold. It is found to be of block-diagonal form

$$
\mathrm{H}=\left(\begin{array}{cc}
\mathrm{H}_{k k} & 0 \\
0 & \mathrm{H}_{\varphi \varphi}
\end{array}\right),
$$

with

$$
\mathrm{H}_{k k}=\frac{1}{2 \kappa_{*}} \mathrm{M}_{r}, \quad \mathrm{H}_{\varphi \varphi}=\frac{k_{*}^{2}}{2 \kappa_{*}} \mathrm{M}_{r} .
$$


Apart from prefactors, both blocks are given by the $2 r \times 2 r$ matrix

$$
\mathrm{M}_{r}=\left(\begin{array}{ccccc}
1 & -(1-\mu) & & -\mu \\
-(1-\mu) & 1 & -\mu & & \\
& -\mu & \ddots & \ddots & \\
-\mu & & \ddots & \ddots & -(1-\mu) \\
& & & -(1-\mu) & 1
\end{array}\right) \text {, }
$$

where all empty entries should be set to zero. Here, $\mu=$ $R_{1} /\left(R_{1}+R_{2}\right)$ and in the limit of the plane-sphere geometry, $R_{2} \rightarrow \infty, \mu$ goes to zero. The off-diagonal matrix elements alternate between $-(1-\mu)$ and $-\mu$ in accordance with the alternating reflection at the two spheres during round trips. In the special case of a single round trip, $r=1$, the two different off-diagonal matrix elements add up to yield

$$
\mathrm{M}_{1}=\left(\begin{array}{cc}
1 & -1 \\
-1 & 1
\end{array}\right)
$$

The eigenvalues of the matrix $\mathrm{M}_{r}$ are found as

$$
\lambda_{ \pm}^{(j)}=1 \pm \sqrt{1-4 \mu(1-\mu) \sin ^{2}\left(\frac{\pi j}{r}\right)}
$$

for $j=0, \ldots, r-1$. Both $\mathrm{H}_{k k}$ and $\mathrm{H}_{\varphi \varphi}$ have a zero eigenvalue corresponding to the saddle-point manifold discussed in the previous subsection. Within the saddle-point approximation, the directions perpendicular to the saddle-point manifold can now be integrated out in the usual way, while the integration along the families has to be carried out exactly. This can be done for $\varphi_{*}$ so that we are left with an integral over $k_{*}$ in the following subsection.

Before turning to the result for the trace over $r$ round-trip operators, we would like to make another comment on the plane-sphere case. Considering the eigenvalues (29) to leading order in $\mu$, we find $r$ eigenvalues satisfying

$$
\left(R_{1}+R_{2}\right) \lambda_{-}^{(j)}=2 R_{1} \sin ^{2}(\pi j / r) \quad(\mu \rightarrow 0)
$$

and $r$ eigenvalues $\lambda_{+}^{(j)}=2$. When multiplied by the prefactor $R_{1}+R_{2}$ in the exponent of (16), we obtain from the latter $2 r$ delta functions in the limit $R_{2} \rightarrow \infty$, thus enforcing the conservation of the wave vector $\mathbf{k}$ for the reflection at a plane, as already indicated in the previous subsection. The remaining integrals are controlled by (30) and lead to the PFA result for the plane-sphere geometry proportional to $R_{1}$. Such a result can also be obtained from the more general expression for the sphere-sphere case derived in the remainder of this section.

\section{Casimir free energy and force}

The evaluation of the saddle-point integral is simplified by first forming products $\lambda_{+}^{(j)} \lambda_{-}^{(j)}$ of the eigenvalues (29) for $j=$ $1, \ldots, r-1$ and noting that

$$
\prod_{j=1}^{r-1} \sin \left(\frac{\pi j}{r}\right)=\frac{r}{2^{r-1}}
$$

Then, the inverse of the square root of all nonvanishing eigenvalues of $\left(R_{1}+R_{2}\right) \mathrm{H}$ is found to read

$$
\left(\prod_{\lambda \neq 0} \lambda\right)^{-1 / 2}=\frac{R_{\mathrm{eff}}}{4 r^{2}} \frac{k_{*}}{\kappa_{*}}\left(\frac{4 \kappa_{*}^{2}}{k_{*}^{2}} \frac{1}{R_{1} R_{2}}\right)^{r},
$$

where we have defined the effective radius

$$
R_{\text {eff }}=\frac{R_{1} R_{2}}{R_{1}+R_{2}} .
$$

Changing to the eigenbasis of $\mathrm{H}$ but keeping $k_{*}$ and $\varphi_{*}$ as variables for the integration, i.e., not normalizing the eigenvectors corresponding to the saddle-point manifolds, yields a factor $2 r$ arising through the Jacobian. Then, by applying the multidimensional saddle-point integration formula, we obtain

$$
\operatorname{tr} \mathcal{M}_{p}^{r} \simeq \frac{R_{\mathrm{eff}}}{2 r} \int_{\left|\xi_{n}\right| / c}^{\infty} d \kappa_{*}\left[r_{p}^{(1)} r_{p}^{(2)} e^{-2 \kappa_{*} L}\right]^{r}
$$

for the two polarization contributions $p=$ TE,TM. Inserting this result into (6), we can evaluate the sum over the number $r$ of round trips and obtain for the free energy

$$
\begin{aligned}
\mathcal{F} \simeq & -\frac{k_{B} T R_{\mathrm{eff}}}{4} \sum_{n=-\infty}^{+\infty} \sum_{p \in\{\mathrm{TE}, \mathrm{TM}\}} \int_{\left|\xi_{n}\right| / c}^{\infty} d \kappa_{*} \\
& \times \mathrm{Li}_{2}\left(r_{p}^{(1)} r_{p}^{(2)} e^{-2 \kappa_{*} L}\right),
\end{aligned}
$$

where $\mathrm{Li}_{2}$ denotes the dilogarithm [55]. The Fresnel coefficients (13) are evaluated at the angle $\theta$ defined in (24) taken at the frequencies $\left|\xi_{n}\right|$.

The Casimir force can now be obtained by taking the negative derivative of the expression (35) for the free energy with respect to the distance $L$. We thus find the Lifshitz formula

$$
F \simeq 2 \pi R_{\text {eff }} \mathcal{F}_{\mathrm{PP}}(L, T),
$$

with the free energy per area for two planes at distance $L$ and temperature $T$

$$
\begin{aligned}
\mathcal{F}_{\mathrm{PP}}(L, T)= & \frac{k_{\mathrm{B}} T}{2} \sum_{n=-\infty}^{+\infty} \sum_{p \in\{\mathrm{TE}, \mathrm{TM}\}} \int_{\left|\xi_{n}\right| / c}^{\infty} \frac{d \kappa}{2 \pi} \kappa \\
& \times \log \left(1-r_{p}^{(1)} r_{p}^{(2)} e^{-2 \kappa L}\right) .
\end{aligned}
$$

It is straightforward to extend this result to the zerotemperature case. As already discussed at the end of the previous subsection, these results are also valid in the planesphere case where $R_{\text {eff }}$ is replaced by the sphere radius.

\section{EFFECTIVE AREA}

The most precise Casimir experiments employ spherical lenses [5] or coated microspheres attached to a cantilever beam $[7,9,16,56]$ instead of whole spherical surfaces. Since the experimental data are analyzed with the help of the PFA, it is important to understand what section of the spherical surface actually contributes to the leading asymptotics. For instance, in the case of a spherical lens, such analysis would define the minimum transverse lens size required for equivalence with a complete spherical surface. Here, we estimate the size of the relevant sphere section and proceed in two steps. First, we employ our saddle-point calculation to estimate the typical 


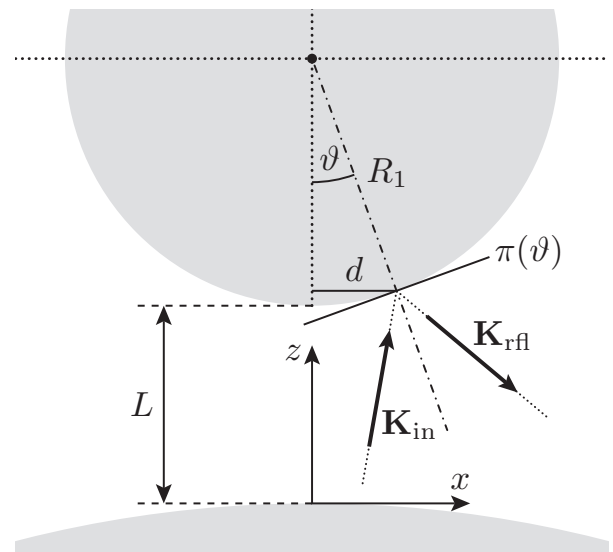

FIG. 3. Estimation of the effective area contributing to the Casimir interaction between two spheres. $\mathbf{K}_{\text {in }}$ and $\mathbf{K}_{\mathrm{rfl}}$ denote the incident and reflected wave vectors, respectively. To be definite, the reflection is shown at the smaller sphere of radius $R_{1}$. Specular reflection at the tangent plane $\pi(\vartheta)$ entails that the projections of $\mathbf{K}_{\text {in }}$ and $\mathbf{K}_{\mathrm{rfl}}$ on $\pi(\vartheta)$ are equal. On the other hand, the wave-vector projections on the $x-y$ plane are generally different: $\delta k=\mid \mathbf{k}_{\mathrm{rfl}}-$ $\mathbf{k}_{\text {in }} \mid \approx 2 \vartheta k_{z}$ for $\vartheta \ll 1$. We can estimate the angular sector effectively contributing to the Casimir interaction from the width $\delta k$ of the Gaussian integrand in the saddle-point approximation (see text).

change in the projection of the wave vector onto the $x-y$ plane during reflection at one of the spheres. Second, we use geometric arguments to obtain the corresponding size of the sphere section in real space.

Even though we first consider the reflection at a single sphere, this reflection is still to be taken in the context of the sphere-sphere setup. Therefore, we keep the saddle-point manifold (20) obtained in Sec. V B. Considering only a single reflection, we denote the incident and reflected wave vectors as $\mathbf{K}_{\text {in }}$ and $\mathbf{K}_{\mathrm{rfl}}$, respectively, as indicated in Fig. 3. For simplicity, we take $\varphi_{\text {in }}=\varphi_{\text {rfl }}=\varphi_{*}$ and concentrate on the modulus of k. From (16) and (18), the Gaussian contribution of a single reflection at a sphere with radius $R_{j}, j=1,2$ can then be identified as

$$
\exp \left(-\eta R_{j}\right)=\exp \left(-\frac{R_{j}}{4 \kappa_{*}}\left(k_{\mathrm{in}}-k_{\mathrm{rfl}}\right)^{2}\right) .
$$

Here, $\eta$ is defined in analogy to (19) with the two wave-vector components replaced by $\kappa_{\text {in }}$ and $\kappa_{\text {rfl }}$.

Neglecting numerical factors of order one, the width around the saddle-point manifold is thus $\delta k^{(j)} \sim \sqrt{\kappa_{*} / R_{j}}$. The typical scale of $\kappa_{*}$ is set by the integral on the right-hand side of (34), finally leading to the change of the projection of the wave vector onto the $x-y$ plane

$$
\delta k^{(j)}=\left|\mathbf{k}_{\mathrm{rfl}}-\mathbf{k}_{\text {in }}\right| \sim\left(L R_{j}\right)^{-1 / 2} .
$$

As expected, the scattering at the smaller sphere provides the larger deviations from the saddle-point manifold. Thus the effective area contributing to the Casimir interaction is fixed by the smaller radius which we refer to as $R_{1}$ in the following.

We now explore the implications of (39) in real space based on specular reflection. The saddle-point manifold (20) corresponds to reflections between the points on the two spheres corresponding to the closest distance $L$ (cf. Fig. 3).
Deviations from the saddle-point manifold as allowed by the Gaussian (38) implicate the surroundings of these two points in the scattering process. We estimate the dimension of the spherical cap on the surface of the smaller sphere 1 by considering the scattering of propagating waves in the real frequency domain with the help of Fig. 3.

As above, we assume $\mathbf{k}_{\mathrm{in}}$ and $\mathbf{k}_{\mathrm{rfl}}$ to be parallel and for simplicity omit the index $j=1$ when writing $\delta k=\left|\mathbf{k}_{\mathrm{rfl}}-\mathbf{k}_{\mathrm{in}}\right|$. As shown in Sec. IV, the WKB approximation for the direct reflection term amounts to a specular reflection at a tangent plane $\pi(\vartheta)$ making an angle $\vartheta$ with the $x-y$ plane. For parallel vectors and small values of $\delta k$, a simple relation between $\vartheta$ and $\delta k$ can be derived by noting that while the projection of the wave vector onto the plane $\pi(\vartheta)$ is conserved during a scattering process, this is not the case for the projection onto the $x-y$ plane for nonvanishing values of $\vartheta$. Assuming $\vartheta \ll 1$, we find

$$
\delta k \approx 2 \vartheta k_{z} .
$$

This relation together with the scaling $k_{z} \sim 1 / L$ allows us to estimate the width of the angular sector effectively contributing to the Casimir interaction from the Gaussian width (39).

We find that the spherical cap around the point of closest distance corresponds to the angular sector bounded by the angle $\vartheta \sim\left(L / R_{1}\right)^{1 / 2} \ll 1$. As indicated by Fig. 3, its transverse size is $d \approx R_{1} \vartheta \sim\left(R_{1} L\right)^{1 / 2} \ll R_{1}<R_{2}$. The same scaling was found by a heuristic geometric argument [57]. The area of the spherical surface effectively contributing to the interaction is then $A \sim R_{1} L$, which coincides, except for a numerical factor of order one, with the ratio between the Casimir force for two spheres within PFA and the Casimir pressure for parallel planes, as long as the interaction obeys a power law [58].

Although the discussion above holds for arbitrary temperatures, we show in the remainder of this section that the effective area for the thermal corrections scales in a different way in the low-temperature regime. The difference arises from the typical scale for $\kappa_{*}$, which is no longer set by $1 / L$, but rather by $1 / \lambda_{T}$, where $\lambda_{T}=\hbar c / k_{B} T$ is the thermal wavelength. In order to illustrate this property, we consider the thermal correction of the Casimir force $\delta F \equiv F(L, T)-F(L, 0)$ as an example. We start from Eqs. (36) and (37) and employ the Poisson summation formula [59] to write

$$
\begin{aligned}
\delta F= & 2 \hbar R_{\mathrm{eff}} \sum_{m=1}^{\infty} \sum_{p} \int_{0}^{\infty} d \xi \cos \left(m \lambda_{T} \xi / c\right) \int_{\xi / c}^{\infty} \frac{d \kappa}{2 \pi} \kappa \\
& \times \log \left(1-r_{p}^{(1)} r_{p}^{(2)} e^{-2 \kappa L}\right) .
\end{aligned}
$$

In the low-temperature limit, $L \ll \lambda_{T}$, the exponential $\exp (-2 \kappa L)$ can be taken to be approximately constant and does not provide a cutoff for the $\kappa$ integration in (41). For instance, in the case of plasma metals, the correction $\delta F$ can be written in terms of simple integrals involving trigonometric functions of $m \lambda_{T} \kappa$, which are similar to the expressions derived for the Casimir pressure between parallel planes in Ref. [59]. The derivation of the low-temperature limit of (41) for Drude metals is more involved [60], but $1 / \lambda_{T}$ also provides the typical scale of $\kappa$ in this case.

As a consequence, the effective area contributing to the thermal correction $\delta F$ is found to be of the order of $A^{(T)} \sim R_{1} \lambda_{T}$ and thus much larger than the area relevant for $F(L, T)$, which 
is dominated by the zero-temperature (vacuum) contribution in the low-temperature limit. This result is consistent with the numerical examples for a scalar field presented in Ref. [61].

The thermal correction to the Casimir force has been measured in the plane-sphere geometry by employing a coated lens with $R_{1}=15.6 \mathrm{~cm}$ [5]. The results were analyzed with the help of the PFA, which can be expected to provide an accurate description of the thermal correction if the transverse size of the lens is much larger than $\sqrt{R_{1} \lambda_{T}} \sim 1 \mathrm{~mm}$. If our estimate valid for $L \ll \lambda_{T}$ applies to the experiment where $L \lesssim 0.4 \lambda_{T}$, we can conclude that the lens was indeed of sufficient size.

In most Casimir force measurements, thermal corrections are typically very small. Nevertheless, our estimation of an enlarged effective area is still relevant for thermodynamic quantities vanishing in the zero-temperature limit, in particular for the Casimir entropy.

\section{CONCLUSIONS}

For two spheres of arbitrary radii, we have derived the proximity force approximation expression for the Casimir free energy as the leading asymptotic result for distances between the spheres much smaller than their radii. We have made use of the WKB Mie scattering amplitudes where only the direct reflection term in the Debye expansion contributes to leading order. The trace over a number of round-trip matrices has been evaluated within the saddle-point approximation. The saddle point corresponds to the conservation of the wave-vector component perpendicular to the line connecting the centers of the two spheres. Therefore, the leading asymptotics results from specular reflection in the vicinity of the points of closest distance between the spheres. As an important consequence, we find that no polarization mixing contributes to leading order. The special case of the plane-sphere geometry is recovered by taking the radius of one sphere to infinity.

Although our approach is based on the momentum representation, we are able to estimate the effective area contributing to the Casimir interaction by making use of the localization principle, which allows us to associate a specific impact parameter to a given scattering angle in the WKB approximation. Taken together, the results presented here show that the PFA regime is governed by local scattering from an area of the order of $R_{1} L$ around the points of closest approach, where $R_{1}$ is the radius of the smaller sphere. On the other hand, for thermal corrections in the low-temperature regime, the area becomes much larger and is of the order of $R_{1} \lambda_{T}$. As not all Casimir experiments make use of whole spheres, these estimations provide a condition on the minimum size of the spherical surface required for the PFA to hold for the sphere-sphere or plane-sphere geometries. From a more theoretical perspective, our results help one to understand why local approaches such as the derivative expansion are capable of providing both the leading and nextto-leading order terms in several situations of interest.

\section{ACKNOWLEDGMENTS}

We thank A. Lambrecht, S. Reynaud, and H. M. Nussenzveig for inspiring discussions. This work has been supported by the Coordination for the Improvement of Higher Education Personnel (CAPES) and the German Academic Exchange Service (DAAD) through the PROBRAL collaboration program.
P.A.M.N. also thanks the Brazilian agencies National Council for Scientific and Technological Development (CNPq), the National Institute of Science and Technology Complex Fluids (INCT-FCx), the Carlos Chagas Filho Foundation for Research Support of Rio de Janeiro (FAPERJ), and the São Paulo Research Foundation (FAPESP).

\section{APPENDIX A: DERIVATION OF THE REFLECTION OPERATOR MATRIX ELEMENTS}

In the sphere-sphere geometry, the axis connecting the centers of the two spheres is distinguished. We have defined it as $z$ axis and used the polarization basis $\left\{\hat{\boldsymbol{\epsilon}}_{\mathrm{TE}}, \hat{\boldsymbol{\epsilon}}_{\mathrm{TM}}\right\}$ taken with respect to the incidence plane as specified in (1). When a plane wave $\left|\mathbf{K}_{i}, p_{i}\right\rangle$ is scattered into a plane wave $\left|\mathbf{K}_{j}, p_{j}\right\rangle$, the relations between the polarization basis vectors can be expressed as

$$
\begin{aligned}
\hat{\boldsymbol{\epsilon}}_{\mathrm{TE}}\left(\mathbf{K}_{i}\right) \cdot \hat{\boldsymbol{\epsilon}}_{\mathrm{TE}}\left(\mathbf{K}_{j}\right) & =\cos (\varphi), \\
\hat{\boldsymbol{\epsilon}}_{\mathrm{TM}}\left(\mathbf{K}_{i}\right) \cdot \hat{\boldsymbol{\epsilon}}_{\mathrm{TM}}\left(\mathbf{K}_{j}\right) & =-\frac{c^{2}}{\xi^{2}}\left[k_{i} k_{j}-\phi_{i} \phi_{j} \kappa_{i} \kappa_{j} \cos (\varphi)\right], \\
\hat{\boldsymbol{\epsilon}}_{\mathrm{TE}}\left(\mathbf{K}_{i}\right) \cdot \hat{\boldsymbol{\epsilon}}_{\mathrm{TM}}\left(\mathbf{K}_{j}\right) & =-\frac{c \phi_{j} \kappa_{j}}{\xi} \sin (\varphi), \\
\hat{\boldsymbol{\epsilon}}_{\mathrm{TM}}\left(\mathbf{K}_{i}\right) \cdot \hat{\boldsymbol{\epsilon}}_{\mathrm{TE}}\left(\mathbf{K}_{j}\right) & =\frac{c \phi_{i} \kappa_{i}}{\xi} \sin (\varphi),
\end{aligned}
$$

where $\varphi=\varphi_{j}-\varphi_{i}$

Another distinguished polarization basis is defined by the scattering plane spanned by the two wave vectors involved in the scattering process. In the corresponding basis with polarization vectors perpendicular and parallel to the scattering plane defined as

$$
\begin{aligned}
\hat{\boldsymbol{\epsilon}}_{\perp}\left(\mathbf{K}_{i}\right) & =\frac{\hat{\mathbf{K}}_{j} \times \hat{\mathbf{K}}_{i}}{\left|\hat{\mathbf{K}}_{j} \times \hat{\mathbf{K}}_{i}\right|}, \\
\hat{\boldsymbol{\epsilon}}_{\|}\left(\mathbf{K}_{i}\right) & =\hat{\boldsymbol{\epsilon}}_{\perp} \times \hat{\mathbf{K}}_{i},
\end{aligned}
$$

respectively, the polarization is conserved during the scattering process. More specifically, we have [52]

$$
\begin{aligned}
\mathcal{R}\left|\mathbf{K}_{i}, \perp\right\rangle & =\frac{2 \pi c}{\xi \kappa_{j}} S_{1}\left|\mathbf{K}_{j}, \perp\right\rangle, \\
\mathcal{R}\left|\mathbf{K}_{i}, \|\right\rangle & =\frac{2 \pi c}{\xi \kappa_{j}} S_{2}\left|\mathbf{K}_{j}, \|\right\rangle,
\end{aligned}
$$

with the scattering amplitudes $S_{1}$ and $S_{2}$ defined in (9). The basis vectors (A2) for the incoming and outgoing wave vectors are related by

$$
\begin{aligned}
\hat{\boldsymbol{\epsilon}}_{\perp}\left(\mathbf{K}_{i}\right) \cdot \hat{\boldsymbol{\epsilon}}_{\perp}\left(\mathbf{K}_{j}\right) & =1, \\
\hat{\boldsymbol{\epsilon}}_{\|}\left(\mathbf{K}_{i}\right) \cdot \hat{\boldsymbol{\epsilon}}_{\|}\left(\mathbf{K}_{j}\right) & =\cos (\Theta), \\
\hat{\boldsymbol{\epsilon}}_{\|}\left(\mathbf{K}_{i}\right) \cdot \hat{\boldsymbol{\epsilon}}_{\perp}\left(\mathbf{K}_{j}\right) & =0, \\
\hat{\boldsymbol{\epsilon}}_{\perp}\left(\mathbf{K}_{i}\right) \cdot \hat{\boldsymbol{\epsilon}}_{\|}\left(\mathbf{K}_{j}\right) & =0 .
\end{aligned}
$$

The coefficients $A, B, C$, and $D$ appearing in (8) reflect the change of polarization basis. They can be expressed as

$$
\begin{aligned}
& A=\left[\hat{\boldsymbol{\epsilon}}_{\mathrm{TE}}\left(\mathbf{K}_{j}\right) \cdot \hat{\boldsymbol{\epsilon}}_{\perp}\left(\mathbf{K}_{j}\right)\right]\left[\hat{\boldsymbol{\epsilon}}_{\mathrm{TE}}\left(\mathbf{K}_{i}\right) \cdot \hat{\boldsymbol{\epsilon}}_{\perp}\left(\mathbf{K}_{i}\right)\right], \\
& B=\left[\hat{\boldsymbol{\epsilon}}_{\mathrm{TM}}\left(\mathbf{K}_{j}\right) \cdot \hat{\boldsymbol{\epsilon}}_{\perp}\left(\mathbf{K}_{j}\right)\right]\left[\hat{\boldsymbol{\epsilon}}_{\mathrm{TM}}\left(\mathbf{K}_{i}\right) \cdot \hat{\boldsymbol{\epsilon}}_{\perp}\left(\mathbf{K}_{i}\right)\right],
\end{aligned}
$$




$$
\begin{aligned}
C & =-\left[\hat{\boldsymbol{\epsilon}}_{\mathrm{TM}}\left(\mathbf{K}_{j}\right) \cdot \hat{\boldsymbol{\epsilon}}_{\perp}\left(\mathbf{K}_{j}\right)\right]\left[\hat{\boldsymbol{\epsilon}}_{\mathrm{TE}}\left(\mathbf{K}_{i}\right) \cdot \hat{\boldsymbol{\epsilon}}_{\perp}\left(\mathbf{K}_{i}\right)\right], \\
D & =\left[\hat{\boldsymbol{\epsilon}}_{\mathrm{TE}}\left(\mathbf{K}_{j}\right) \cdot \hat{\boldsymbol{\epsilon}}_{\perp}\left(\mathbf{K}_{j}\right)\right]\left[\hat{\boldsymbol{\epsilon}}_{\mathrm{TM}}\left(\mathbf{K}_{i}\right) \cdot \hat{\boldsymbol{\epsilon}}_{\perp}\left(\mathbf{K}_{i}\right)\right] .
\end{aligned}
$$

Alternative expressions can be obtained by means of the relations

$$
\begin{aligned}
\hat{\boldsymbol{\epsilon}}_{\mathrm{TE}} \cdot \hat{\boldsymbol{\epsilon}}_{\|} & =-\hat{\boldsymbol{\epsilon}}_{\mathrm{TM}} \cdot \hat{\boldsymbol{\epsilon}}_{\perp}, \\
\hat{\boldsymbol{\epsilon}}_{\mathrm{TM}} \cdot \hat{\boldsymbol{\epsilon}}_{\|} & =\hat{\boldsymbol{\epsilon}}_{\mathrm{TE}} \cdot \hat{\boldsymbol{\epsilon}}_{\perp} .
\end{aligned}
$$

Expressing the scalar products (A1) in terms of the polarization basis $\left\{\hat{\boldsymbol{\epsilon}}_{\perp}, \hat{\boldsymbol{\epsilon}}_{\|}\right\}$, we find the relations

$$
\begin{aligned}
\hat{\boldsymbol{\epsilon}}_{\mathrm{TE}}\left(\mathbf{K}_{i}\right) \cdot \hat{\boldsymbol{\epsilon}}_{\mathrm{TE}}\left(\mathbf{K}_{j}\right) & =A+B \cos (\Theta), \\
\hat{\boldsymbol{\epsilon}}_{\mathrm{TM}}\left(\mathbf{K}_{i}\right) \cdot \hat{\boldsymbol{\epsilon}}_{\mathrm{TM}}\left(\mathbf{K}_{j}\right) & =A \cos (\Theta)+B, \\
\hat{\boldsymbol{\epsilon}}_{\mathrm{TE}}\left(\mathbf{K}_{i}\right) \cdot \hat{\boldsymbol{\epsilon}}_{\mathrm{TM}}\left(\mathbf{K}_{j}\right) & =-C-D \cos (\Theta), \\
\hat{\boldsymbol{\epsilon}}_{\mathrm{TM}}\left(\mathbf{K}_{i}\right) \cdot \hat{\boldsymbol{\epsilon}}_{\mathrm{TE}}\left(\mathbf{K}_{j}\right) & =C \cos (\Theta)+D .
\end{aligned}
$$

Solving for the coefficients $A, B, C$, and $D$ and making use of (A1), we finally obtain

$$
\begin{aligned}
A\left(\mathbf{K}_{i}, \mathbf{K}_{j}\right) & =\frac{\xi^{4} \cos (\varphi)-c^{4}\left[k_{i} k_{j} \cos (\varphi)-\phi_{i} \phi_{j} \kappa_{i} \kappa_{j}\right]\left[k_{i} k_{j}-\phi_{i} \phi_{j} \kappa_{i} \kappa_{j} \cos (\varphi)\right]}{\xi^{4}-c^{4}\left[k_{i} k_{j} \cos (\varphi)-\phi_{i} \phi_{j} \kappa_{i} \kappa_{j}\right]^{2}}, \\
B\left(\mathbf{K}_{i}, \mathbf{K}_{j}\right) & =-\frac{\xi^{2} c^{2} k_{i} k_{j} \sin ^{2}(\varphi)}{\xi^{4}-c^{4}\left[k_{i} k_{j} \cos (\varphi)-\phi_{i} \phi_{j} \kappa_{i} \kappa_{j}\right]^{2}}, \\
C\left(\mathbf{K}_{i}, \mathbf{K}_{j}\right) & =c^{3} \xi \sin (\varphi) \frac{k_{i} k_{j} \phi_{i} \kappa_{i} \cos (\varphi)-k_{i}^{2} \phi_{j} \kappa_{j}}{\xi^{4}-c^{4}\left[k_{i} k_{j} \cos (\varphi)-\phi_{i} \phi_{j} \kappa_{i} \kappa_{j}\right]^{2}}, \\
D\left(\mathbf{K}_{i}, \mathbf{K}_{j}\right) & =C\left(-\mathbf{K}_{j},-\mathbf{K}_{i}\right) .
\end{aligned}
$$

For $\xi=0$, they simplify to

$$
A=-\phi_{i} \phi_{j}, \quad B=C=D=0,
$$

and for $\mathbf{k}_{i}=\mathbf{k}_{j}$ we find

$$
A=1, \quad B=C=D=0 .
$$

The matrix elements of the reflection operators (8) are not all mutually independent since they fulfill reciprocity relations [62-64]. In our notation these relations read

$$
\kappa_{i}\left\langle\mathbf{K}_{i}, p_{i}|\mathcal{R}| \mathbf{K}_{j}, p_{j}\right\rangle=\kappa_{j}(-1)^{p_{i}+p_{j}}\left\langle-\mathbf{K}_{j}, p_{j}|\mathcal{R}|-\mathbf{K}_{i}, p_{i}\right\rangle,
$$

where $(-1)^{p_{i}+p_{j}}$ is +1 if the polarizations $p_{i}$ and $p_{j}$ are equal and -1 otherwise. Indeed, since

$$
\begin{aligned}
& A\left(\mathbf{K}_{i}, \mathbf{K}_{j}\right)=A\left(-\mathbf{K}_{j},-\mathbf{K}_{i}\right), \\
& B\left(\mathbf{K}_{i}, \mathbf{K}_{j}\right)=B\left(-\mathbf{K}_{j},-\mathbf{K}_{i}\right),
\end{aligned}
$$

it is straightforward to verify that the coefficients (A8) satisfy the reciprocity relations.

\section{APPENDIX B: LOW-FREQUENCY LIMIT OF THE SCATTERING AMPLITUDES}

The WKB approximation for the scattering amplitudes (12) at a sphere discussed in Sec. IV has been derived for large size parameters $\xi R / c$. As a consequence, the zero-frequency contribution in the Matsubara sum (4) is a priori not covered by (12).

By analyzing the low-frequency limit of the scattering amplitudes, we will demonstrate that the scattering amplitudes (12) and the matrix elements (8) obtained from them can be employed even in the limit of zero frequency. At this point, it is worth noting that even though the scattering amplitudes (12) vanish in the limit $\xi \rightarrow 0$, this is not the case for the matrix elements (8). Therefore, we need to keep terms linear in $\xi$ in the low-frequency expression for the scattering amplitudes.
In the following, we will distinguish three classes of materials: perfect reflectors, real metals with a finite dc conductivity, and dielectrics. For perfect reflectors the permittivity is infinite for all frequencies, comprising also the plasma model when the sphere radius is much larger than the plasma wavelength. Real metals exhibit a finite permittivity except in the zero-frequency limit where the finite dc conductivity gives rise to a divergence proportional to $1 / \xi$. Finally, for dielectrics, the permittivity remains finite for $\xi \rightarrow 0$.

We start from the expressions (9) for the Mie scattering amplitudes and first consider the material-independent functions $\pi_{\ell}$ and $\tau_{\ell}$. Noting that, according to $(10), \cos (\Theta)$ at low frequencies diverges like $1 / \xi^{2}$, we find the dominant low-frequency behavior

$$
\begin{aligned}
\pi_{\ell}(\cos (\Theta)) & \simeq \frac{(2 \ell) !}{2^{\ell}(\ell-1) ! \ell !} \cos ^{\ell-1}(\Theta) \sim \frac{1}{\xi^{2 \ell-2}}, \\
\tau_{\ell}(\cos (\Theta)) & \simeq \frac{(2 \ell) !}{2^{\ell}[(\ell-1) !]^{2}} \cos ^{\ell}(\Theta) \sim \frac{1}{\xi^{2 \ell}} .
\end{aligned}
$$

As a consequence, among the four combinations of these two functions and the two Mie coefficients in (9), only those involving $\tau_{\ell}$ can potentially lead to contributions linear in $\xi$. Terms involving $\pi_{\ell}$ yield an additional factor $\xi^{2}$ and can thus be disregarded. Furthermore, only Mie coefficients going like $\xi^{2 \ell+1}$ can then lead to a relevant contribution to the scattering amplitudes.

Such a behavior is found for the Mie coefficient $a_{\ell}$ for which the leading term at low frequencies can be expressed as

$$
a_{\ell} \simeq(-1)^{\ell} \frac{(\ell+1)(\ell !)^{2}}{2 \ell(2 \ell+1)[(2 \ell) !]^{2}} E_{\ell}^{\mathrm{mat}}\left(\frac{2 \xi R}{c}\right)^{2 \ell+1} .
$$

The material dependence is contained in the factor $E_{\ell}^{\text {mat }}$ (see [65] for a detailed discussion). For dielectrics, one finds

$$
E_{\ell}^{\text {diel }}=\frac{\varepsilon(0)-1}{\varepsilon(0)+\frac{\ell+1}{\ell}},
$$


while $E_{\ell}^{\text {mat }}=1$ for real metals and perfect reflectors. For the Mie coefficient $b_{\ell}$, the required frequency dependence is only found for perfect reflectors where

$$
b_{\ell} \sim-\frac{\ell}{\ell+1} a_{\ell} .
$$

In contrast, the low-frequency behavior of the Mie coefficient $b_{\ell}$ for real metals is of order $\xi^{2 \ell+2}$ and for dielectrics of order $\xi^{2 \ell+3}$.

Let us now first consider the scattering amplitude $S_{1}(\Theta)$ which according to the preceding analysis is only nonvanishing for perfect metals. This finding is in agreement with the fact that for zero frequency the reflection coefficient for TE modes vanishes for metals with finite dc conductivity and dielectrics. Inserting the Mie coefficient $b_{\ell}$ and the function $\tau_{\ell}(\cos \Theta)$ for perfect metals, we obtain

$$
S_{1}(\Theta) \simeq-\frac{\xi R}{c} \sum_{\ell=1}^{\infty} \frac{\ell}{\ell+1} \frac{\left[\frac{R}{c}\left(-2 \xi^{2} \cos (\Theta)\right)^{1 / 2}\right]^{2 \ell}}{(2 \ell) !}
$$

We recall that in the matrix elements (8) of the reflection operator this function appears together with a prefactor $\xi^{-1}$.
Furthermore, according to $(10), \xi^{2} \cos (\Theta)$ does not vanish in the limit $\xi \rightarrow 0$. For large radius $R$, we then find

$$
S_{1}(\Theta) \simeq-\frac{\xi R}{2 c} e^{2(\xi R / c) \sin (\Theta / 2)}
$$

where we made use of the relation

$$
[-2 \cos (\Theta)]^{1 / 2}=2 \sin (\Theta / 2)
$$

valid for $\xi=0$. For perfect reflectors, where $r_{\mathrm{TE}}=-1$, Eq. (B7) agrees with the WKB result (12).

The validity of the WKB approximation of $S_{2}(\Theta)$ can be proven along the same lines, observing that, for perfect reflectors and real metals, $r_{\mathrm{TM}}=1$ at zero frequency. For large $R$, the dominant contribution to the scattering amplitudes arises from large angular momenta $\ell$. Therefore, $E_{\ell}^{\text {diel }}$ can be replaced by $[\varepsilon(0)-1] /[\varepsilon(0)+1]$, which according to (13) agrees with $r_{\mathrm{TM}}$ in the low-frequency limit. This completes the proof of the applicability of (12) even in the limit $\xi \rightarrow 0$.
[1] H. B. G. Casimir, On the attraction between two perfectly conducting plates, Proc. K. Ned. Akad. Wet. 51, 793 (1948).

[2] M. Bordag, G. L. Klimchitskaya, U. Mohideen, and V. M. Mostepanenko, Advances in the Casimir Effect (Oxford University Press, Oxford, 2009).

[3] M. J. Sparnaay, Measurements of attractive forces between flat plates, Physica 24, 751 (1958).

[4] G. Bressi, G. Carugno, R. Onofrio, and G. Ruoso, Measurement of the Casimir Force Between Parallel Metallic Surfaces, Phys. Rev. Lett. 88, 041804 (2002).

[5] A. O. Sushkov, W. J. Kim, D. A. R. Dalvit, and S. K. Lamoreaux, Observation of the thermal Casimir force, Nat. Phys. 7, 230 (2011).

[6] G. Torricelli, I. Pirozhenko, S. Thornton, A. Lambrecht, and C. Binns, Casimir force between a metal and a semimetal, Europhys. Lett. 93, 51001 (2011).

[7] C.-C. Chang, A. A. Banishev, R. Castillo-Garza, G. L. Klimchitskaya, V. M. Mostepanenko, and U. Mohideen, Gradient of the Casimir force between Au surfaces of a sphere and a plate measured using an atomic force microscope in a frequency-shift technique, Phys. Rev. B 85, 165443 (2012).

[8] D. Garcia-Sanchez, K. Y. Fong, H. Bhaskaran, S. Lamoreaux, and H. X. Tang, Casimir Force and In Situ Surface Potential Measurements on Nanomembranes, Phys. Rev. Lett. 109, 027202 (2012).

[9] A. A. Banishev, G. L. Klimchitskaya, V. M. Mostepanenko, and U. Mohideen, Demonstration of the Casimir Force Between Ferromagnetic Surfaces of a Ni-Coated Sphere and a Ni-Coated Plate, Phys. Rev. Lett. 110, 137401 (2013).

[10] M. Sedighi, V. B. Svetovoy, and G. Palasantzas, Casimir force measurements from silicon carbide surfaces, Phys. Rev. B 93, 085434 (2016).

[11] G. Bimonte, D. López, and R. S. Decca, Isoelectronic determination of the thermal Casimir force, Phys. Rev. B 93, 184434 (2016).
[12] G. L. Klimchitskaya, U. Mohideen, and V. M. Mostepanenko, The Casimir force between real materials: Experiment and theory, Rev. Mod. Phys. 81, 1827 (2009).

[13] R. Decca, V. Aksyuk, and D. López, Casimir force in micro and nano electro mechanical systems, Lect. Notes Phys. 834, 287 (2011).

[14] S. K. Lamoreaux, Progress in experimental measurements of the surface-surface Casimir force: Electrostatic calibrations and limitations to accuracy, Lect. Notes Phys. 834, 219 (2011).

[15] D. S. Ether, Jr. et al., Probing the Casimir force with optical tweezers, Europhys. Lett. 112, 44001 (2015).

[16] J. L. Garrett, D. A. T. Somers, and J. N. Munday, Measurement of the Casimir Force Between Two Spheres, Phys. Rev. Lett. 120, 040401 (2018).

[17] A. Lambrecht, P. A. Maia Neto, and S. Reynaud, The Casimir effect within scattering theory, New J. Phys. 8, 243 (2006).

[18] T. Emig, N. Graham, R. L. Jaffe, and M. Kardar, Casimir Forces Between Arbitrary Compact Objects, Phys. Rev. Lett. 99, 170403 (2007).

[19] S. J. Rahi, T. Emig, N. Graham, R. L. Jaffe, and M. Kardar, Scattering theory approach to electrodynamic Casimir forces, Phys. Rev. D 80, 085021 (2009).

[20] M. T. Jaekel and S. Reynaud, Casimir force between partially transmitting mirrors, J. Phys. I 1, 1395 (1991).

[21] C. Genet, A. Lambrecht, and S. Reynaud, Casimir force and the quantum theory of lossy optical cavities, Phys. Rev. A 67, 043811 (2003).

[22] M. Hartmann, G.-L. Ingold, and P. A. Maia Neto, Plasma versus Drude Modeling of the Casimir Force: Beyond the Proximity Force Approximation, Phys. Rev. Lett. 119, 043901 (2017).

[23] M. Hartmann, G.-L. Ingold, and P. A. Maia Neto, Advancing numerics for the Casimir effect to experimentally relevant aspect ratios, arXiv:1803.05791.

[24] G. Bimonte, Going beyond PFA: A precise formula for the sphere-plate Casimir force, Europhys. Lett. 118, 20002 (2017). 
[25] B. Derjaguin, Untersuchungen über die Reibung und Adhäsion, IV - Theorie des Anhaftens kleiner Teilchen, Kolloid-Zs. 69, 155 (1934).

[26] H.-J. Butt and M. Kappl, Surface and Interfacial Forces (WileyVCH Verlag, Weinheim, 2010).

[27] M. Borkovec, I. Szilagyi, I. Popa, M. Finessi, P. Sinha, P. Maroni, and G. Papastavrou, Investigating forces between charged particles in the presence of oppositely charged polyelectrolytes with the multi-particle colloidal probe technique, Adv. Colloid Interface Sci. 179-182, 85 (2012).

[28] M. Elzbieciak-Wodka, M. N. Popescu, F. J. M. Ruiz-Cabello, G. Trefalt, P. Maroni, and M. Borkovec, Measurements of dispersion forces between colloidal latex particles with the atomic force microscope and comparison with Lifshitz theory, J. Chem. Phys. 140, 104906 (2014).

[29] P. A. Maia Neto, A. Lambrecht, and S. Reynaud, Casimir effect with rough metallic mirrors, Phys. Rev. A 72, 012115 (2005).

[30] C. D. Fosco, F. C. Lombardo, and F. D. Mazzitelli, Derivativeexpansion approach to the interaction between close surfaces, Phys. Rev. A 89, 062120 (2014).

[31] C. D. Fosco, F. C. Lombardo, and F. D. Mazzitelli, Proximity force approximation for the Casimir energy as a derivative expansion, Phys. Rev. D 84, 105031 (2011).

[32] G. Bimonte, T. Emig, R. L. Jaffe, and M. Kardar, Casimir forces beyond the proximity approximation, Europhys. Lett. 97, 50001 (2012).

[33] G. Bimonte, T. Emig, and M. Kardar, Material dependence of Casimir forces: Gradient expansion beyond proximity, Appl. Phys. Lett. 100, 074110 (2012).

[34] G. Bimonte, Beyond-proximity-force-approximation Casimir force between two spheres at finite temperature, Phys. Rev. D 97, 085011 (2018).

[35] C. D. Fosco, F. C. Lombardo, and F. D. Mazzitelli, Derivative expansion for the electromagnetic Casimir free energy at high temperatures, Phys. Rev. D 92, 125007 (2015).

[36] M. Bordag and V. Nikolaev, First analytic correction beyond the proximity force approximation in the Casimir effect for the electromagnetic field in sphere-plane geometry, Phys. Rev. D 81, 065011 (2010).

[37] L. P. Teo, M. Bordag, and V. Nikolaev, Corrections beyond the proximity force approximation, Phys. Rev. D 84, 125037 (2011).

[38] L. P. Teo, Material dependence of Casimir interaction between a sphere and a plate: First analytic correction beyond proximity force approximation, Phys. Rev. D 88, 045019 (2013).

[39] A. Bulgac, P. Magierski, and A. Wirzba, Scalar Casimir effect between Dirichlet spheres or a plate and a sphere, Phys. Rev. D 73, 025007 (2006).

[40] M. Bordag and V. Nikolaev, Casimir force for a sphere in front of a plane beyond proximity force approximation, J. Phys. A: Math. Theor. 41, 164002 (2008).

[41] G. Bimonte, Classical Casimir interaction of a perfectly conducting sphere and plate, Phys. Rev. D 95, 065004 (2017).

[42] G. Bimonte and T. Emig, Exact Results for Classical Casimir Interactions: Dirichlet and Drude Model in the Sphere-Sphere and Sphere-Plane Geometry, Phys. Rev. Lett. 109, 160403 (2012).

[43] P. Rodriguez-Lopez, Casimir energy and entropy in the spheresphere geometry, Phys. Rev. B 84, 075431 (2011).
[44] S. Umrath, M. Hartmann, G.-L. Ingold, and P. A. Maia Neto, Disentangling geometric and dissipative origins of negative Casimir entropies, Phys. Rev. E 92, 042125 (2015).

[45] L. P. Teo, Casimir effect between two spheres at small separations, Phys. Rev. D 85, 045027 (2012).

[46] H. M. Nussenzveig, High-frequency scattering by a transparent sphere. I. Direct reflection and transmission, J. Math. Phys. 10, 82 (1969).

[47] H. M. Nussenzveig, Diffraction Effects in Semiclassical Scattering (Cambridge University Press, Cambridge, UK, 1992).

[48] M. Schaden and L. Spruch, Infinity-free semiclassical evaluation of Casimir effects, Phys. Rev. A 58, 935 (1998).

[49] R. L. Jaffe and A. Scardicchio, Casimir Effect and Geometrical Optics, Phys. Rev. Lett. 92, 070402 (2004).

[50] A. Scardicchio and R. L. Jaffe, Casimir effects: An optical approach I. Foundations and examples, Nucl. Phys. B 704, 552 (2005).

[51] M. Nieto-Vesperinas, Scattering and Diffraction in Physical Optics (World Scientific, Singapore, 2006).

[52] C. F. Bohren and D. R. Huffman, Absorption and Scattering of Light by Small Particles (Wiley, New York, 1983), Chap. 4.

[53] L. D. Landau, E. M. Lifshitz, and L. P. Pitaevskii, Electrodynamics of Continuous Media (Pergamon, Oxford, 1984).

[54] H. M. Nussenzveig, High-frequency scattering by an impenetrable sphere, Ann. Phys. (NY) 34, 23 (1965).

[55] NIST Digital Library of Mathematical Functions, http://dlmf.nist.gov/25.12, release 1.0 .16 , edited by F. W. J. Olver, A. B. Olde Daalhuis, D. W. Lozier, B. I. Schneider, R. F. Boisvert, C. W. Clark, B. R. Miller, and B. V. Saunders.

[56] R. S. Decca, D. López, E. Fischbach, G. L. Klimchitskaya, D. E. Krause, and V. M. Mostepanenko, Tests of new physics from precise measurements of the Casimir pressure between two goldcoated plates, Phys. Rev. D 75, 077101 (2007).

[57] W. J. Kim, A. O. Sushkov, D. A. R. Dalvit, and S. K. Lamoreaux, Surface contact potential patches and Casimir force measurements, Phys. Rev. A 81, 022505 (2010).

[58] Since we have neglected numerical factors of order one, we are unable to distinguish between the smallest radius $R_{1}$ and the effective radius $R_{\text {eff }}$ defined in (33), given that their ratio is bounded by $1<R_{1} / R_{\text {eff }} \leqslant 2$.

[59] C. Genet, A. Lambrecht, and S. Reynaud, Temperature dependence of the Casimir effect between metallic mirrors, Phys. Rev. A 62, 012110 (2000).

[60] G.-L. Ingold, A. Lambrecht, and S. Reynaud, Quantum dissipative Brownian motion and the Casimir effect, Phys. Rev. E 80, 041113 (2009).

[61] A. Weber and H. Gies, Nonmonotonic Thermal Casimir Force from Geometry-Temperature Interplay, Phys. Rev. Lett. 105, 040403 (2010).

[62] R. Carminati, M. Nieto-Vesperinas, and J.-J. Greffet, Reciprocity of evanescent electromagnetic waves, J. Opt. Soc. Am. A 15, 706 (1998).

[63] R. Messina and M. Antezza, Scattering-matrix approach to Casimir-Lifshitz force and heat transfer out of thermal equilibrium between arbitrary bodies, Phys. Rev. A 84, 042102 (2011).

[64] R. Messina, P. A. Maia Neto, B. Guizal, and M. Antezza, Casimir interaction between a sphere and a grating, Phys. Rev. A 92, 062504 (2015).

[65] A. Canaguier-Durand, Ph.D. thesis, Université Pierre et Marie Curie, 2011 